\title{
Bloch-Messiah decomposition and Magnus expansion for parametric down-conversion with monochromatic pump: role of the gain
}

\author{
Tobias Lipfert ${ }^{1, *}$, Dmitri Horoshko ${ }^{1,2}$, Giuseppe Patera ${ }^{1}$, and Mikhail Kolobov ${ }^{1}$ \\ ${ }^{1}$ Univ. Lille, CNRS, UMR 8523 - PhLAM - Physique des Lasers Atomes et Molécules, F-59000 Lille, \\ France \\ ${ }^{2}$ B. I. Stepanov Institute of Physics, NASB, Nezavisimosti Ave. 68, Minsk 220072 Belarus
}

\begin{abstract}
We consider the effect of different orders of Magnus expansion for the field transformation in type-I parametric down-conversion with a monochromatic pump. The exact solution, existing in this case, allows us to analyze the convergence of the Magnus expansion for the spectrum of squeezing and the angle of squeezing. We demonstrate how the convergence of the Magnus series depends on the parametric gain for various values of the phase mismatch. For each phase-mismatch angle we find the gain, which is the exact upper bound for the convergence of the Magnus series.
\end{abstract}

\section{Introduction}

Squeezed states of light play a prominent role in modern quantum optics, in particular, providing excellent opportunities for increasing the sensitivity of interferometric measurements and for realisation of continuous-variable protocols of quantum information [1]. The continuouswave narrow-band squeezed light generated by type-I parametric down-conversion (PDC) is perhaps the best-known realization of squeezing in optics. Recent experiments reach $15 \mathrm{~dB}$ of squeezing in a band of roughly $100 \mathrm{MHz}$ [2]. On the other hand, PDC in the pulsed regime $[3,4]$ can reach higher peak degree of squeezing and much higher squeezing bandwidth [5]. However, for a sufficiently high gain the operator ordering becomes important for finding the solution of the field evolution equation in the nonlinear crystal [6-9].

The role of operator ordering in PDC can be naturally studied in the framework of the Magnus expansion of a $T$-ordered exponential $[10,11]$. Neglecting the ordering corresponds to the first order of the Magnus series, which is enough for an approximate description of squeezed light for moderate squeezing, below $12 \mathrm{~dB}[6,12]$. At higher squeezing higher orders of the series are necessary for a proper description of the field state. Recently a general treatment of the Magnus expansion for PDC with a monochromatic pump has been undertaken [12], which allowed one to analyze the convergence of the Magnus series to the exact analytic solution existing in this particular case. In the present work we develop further the approach of Ref. [12] and analyze explicitly the dependence of the squeezing spectrum and the squeezing angle on the parametric gain. We demonstrate the curious fact that the contribution of the higher orders of the Magnus expansion is important for intermediate phasemismatch angle only, being zero at the perfect phase-matching and negligible at a large phase

\footnotetext{
*e-mail: Tobias.Lipfert@univ-lille.fr
} 
mismatch. Besides, we find the exact upper bound in gain for the convergence of the Magnus series as a function of phase-mismatch angle and show that for certain angles it is much higher than the value $g=\pi$, obtained in Ref. [12] from the convergence condition in spectral norm [11].

\section{Magnus expansion for continuous-wave PDC}

We consider the process of collinear type-I PDC in a nonlinear $\chi^{(2)}$ crystal with a planewave monochromatic pump of frequency $\omega_{p}$. We shall assume that the pump wave is strong enough and is undepleted. A coordinate system is chosen with the $z$-axis in the direction of the pump wave propagation and with the origin at the front edge of the crystal. The pump is considered as a classical monochromatic wave $E_{p}^{(+)}(t, z)=E_{p} e^{i\left(k_{p} z-\omega_{p} t\right)}$, with the amplitude $E_{p}$, the wave vector $k_{p}$, and the frequency $\omega_{p}$. The down-converted wave is collinear with the pump wave but has a broadband spectrum of frequencies $\Omega$ around the central frequency $\omega_{0}=\omega_{p} / 2$, with the corresponding wave vector $k_{0}$. The down-converted wave is described by the positive-frequency operator $E^{(+)}(t, z)$ normalized to photon-flux units, which can be decomposed into Fourier components as

$$
E^{(+)}(t, z)=\frac{1}{2 \pi} \int d \Omega a(\Omega, z) e^{i k_{0} z-i\left(\omega_{0}+\Omega\right) t}
$$

where $a(\Omega, z)$ is the annihilation operator of the sideband component at frequency $\omega_{0}+\Omega$ and $k_{0}$ is the wave vector at the frequency $\omega_{0}$. Additionally we consider the slowly varying amplitude operator

$$
\epsilon(\Omega, z)=e^{i\left[k_{0}-k(\Omega)\right] z} a(\Omega, z),
$$

where $k(\Omega)$ is the wave vector of the down-converted light inside the crystal at frequency $\omega_{0}+\Omega$. Both operators fulfill canonical commutation relations

$$
\left[a(\Omega, z), a^{\dagger}\left(\Omega^{\prime}, z\right)\right]=\left[\epsilon(\Omega, z), \epsilon^{\dagger}\left(\Omega^{\prime}, z\right)\right]=2 \pi \delta\left(\Omega-\Omega^{\prime}\right) .
$$

It is well known that in PDC with monochromatic pump the wave at frequency $\omega_{0}+\Omega$ is coupled only to the wave at frequency $\omega_{0}-\Omega$ [13]. For compact description of the field evolution we introduce the four-component column vector

$$
\boldsymbol{\xi}(z)=\left(\begin{array}{c}
\epsilon(\Omega, z) \\
\epsilon(-\Omega, z) \\
\epsilon^{\dagger}(\Omega, z) \\
\epsilon^{\dagger}(-\Omega, z)
\end{array}\right)
$$

and a $4 \times 4$ coupling matrix

$$
\mathbf{F}(z)=\left(\begin{array}{cc}
0 & i \sigma e^{i \Delta(\Omega) z} P \\
i \sigma^{*} e^{-i \Delta(\Omega) z} P & 0
\end{array}\right),
$$

where $\sigma$ is the coupling constant, $\Delta(\Omega)=k(\Omega)+k(-\Omega)-2 k_{0}$ is the phase-mismatch function and

$$
P=\left(\begin{array}{ll}
0 & 1 \\
1 & 0
\end{array}\right)
$$


In the matrix form the equation for the field in the crystal has a simple form [13]

$$
\partial_{z} \boldsymbol{\xi}(z)=-i \mathbf{F}(z) \boldsymbol{\xi}
$$

The formal solution to this equation can be written as $\boldsymbol{\xi}(L)=\mathbf{S} \boldsymbol{\xi}(0)$ with the symplectic matrix

$$
\mathbf{S}=\mathcal{T} \exp \left(-i \int_{0}^{L} d z \mathbf{F}(z)\right)
$$

where $L$ is the crystal length and $\mathcal{T}$ denotes the $z$-ordering operator that sorts matrices with higher $z$ to the right in the Taylor expansion of the exponential. Magnus showed $[10,11]$ that this ordered exponential can be represented as an expansion

$$
\mathbf{S}=e^{\boldsymbol{\Omega}_{1}+\boldsymbol{\Omega}_{2}+\boldsymbol{\Omega}_{3}+\ldots},
$$

where the two first terms are

$$
\mathbf{\Omega}_{1}=-i \int_{0}^{L} d z \mathbf{F}(z), \quad \mathbf{\Omega}_{2}=-\frac{1}{2} \int_{0}^{L} d z_{1} \int_{0}^{z_{1}} d z_{2}\left[\mathbf{F}\left(z_{1}\right), \mathbf{F}\left(z_{2}\right)\right] .
$$

Different orders of the Magnus approximation (MA) are then obtained by truncating the series as, e.g., $\mathbf{S}_{1}=e^{\boldsymbol{\Omega}_{1}}$ in the first order, $\mathbf{S}_{2}=e^{\boldsymbol{\Omega}_{1}+\boldsymbol{\Omega}_{2}}$ in the second one, etc. To simplify the notation we denote the analytical solution with index 0 , i.e., $\mathbf{S}_{0}$.

The slowly varying amplitude operators $\boldsymbol{\xi}(z)$ are connected to the sideband operators $\mathbf{a}(z)=\left(a(\Omega, z), a(-\Omega, z), a^{\dagger}(\Omega, z), a^{\dagger}(-\Omega, z)\right)$ via a diagonal phase shift as $\mathbf{a}(z)=\boldsymbol{\Phi}_{z} \boldsymbol{\xi}(z)$, where

$$
\boldsymbol{\Phi}_{z}=\operatorname{diag}\left\{e^{i\left[k(\Omega)-k_{0}\right] z}, e^{i\left[k(-\Omega)-k_{0}\right] z}, e^{-i\left[k(\Omega)-z_{0}\right] z}, e^{-i\left[k(-\Omega)-k_{0}\right] z}\right\}
$$

such that $\mathbf{a}_{k}(L)=\tilde{\mathbf{S}}_{k} \mathbf{a}_{k}(0)$, where $\tilde{\mathbf{S}}_{k}=\boldsymbol{\Phi}_{L} \mathbf{S}_{k}$.

In the $k$ th order of MA the symplectic transformation matrix $\tilde{\mathbf{S}}_{k}$ can be represented by its Bloch-Messiah decomposition [14] $\tilde{\mathbf{S}}_{k}=\mathbf{V}_{k} \mathbf{D}_{k} \mathbf{W}_{k}^{\dagger}$. In this decomposition the unitary matrices

$$
\begin{aligned}
\mathbf{V}_{k} & =\left(\begin{array}{cc}
V_{k} & 0 \\
0 & V_{k}^{*}
\end{array}\right) \text { with } V_{k}=\frac{e^{i \psi_{L, k}}}{\sqrt{2}}\left(\begin{array}{cc}
e^{i \kappa} & 0 \\
0 & e^{-i \kappa}
\end{array}\right)\left(\begin{array}{cc}
1 & i \\
1 & -i
\end{array}\right), \\
\mathbf{W}_{k} & =\left(\begin{array}{cc}
W_{k} & 0 \\
0 & W_{k}^{*}
\end{array}\right) \text { with } W_{k}=\frac{e^{i \psi_{0, k}}}{\sqrt{2}}\left(\begin{array}{cc}
1 & i \\
1 & -i
\end{array}\right)
\end{aligned}
$$

define respectively the input and output squeezing eigenmodes and the matrix

$$
\mathbf{D}=\left(\begin{array}{ll}
\cosh \left(r_{k}\right) I & \sinh \left(r_{k}\right) I \\
\sinh \left(r_{k}\right) I & \cosh \left(r_{k}\right) I
\end{array}\right)
$$

where $I$ is the $2 \times 2$ identity matrix, defines the degree of squeezing of both squeezing eigenmodes at given detuning $\Omega$. The input-output relation is thus determined by four parameters $r_{k}, \kappa, \psi_{0, k}$ and $\psi_{L, k}$. The parameter $\kappa$ is determined by linear dispersion of the crystal and therefore independent of the gain and the MA order. As shown in Ref. [12], the phase of the pump can be chosen so that $\psi_{0, k}=-\psi_{L, k}$. Note that this symmetry is broken in more general cases of PDC, e.g. for ultrabroadband PDC in aperiodically poled crystals [15, 16]. For the considered here scenario of PDC we can perform a complete analysis of the MA in terms of the squeezing parameter $r_{k}$ and the squeezing angle $\psi_{L, k}$. Below we analyze how these two parameters depend on the gain exponent $g=|\sigma| L$ and the phase-mismatch angle $\theta=\Delta L / 2$. These dimensionless variables facilitate the general discussion whose validity is independent of the crystal configuration. 


\section{Role of the parametric gain}

Let us consider the behavior of the two introduced above parameters at different orders of the parametric gain in terms of the gain exponent $g=|\sigma| L$. In Fig. 1 the two parameters are compared for the three first orders of MA at three different gain levels.
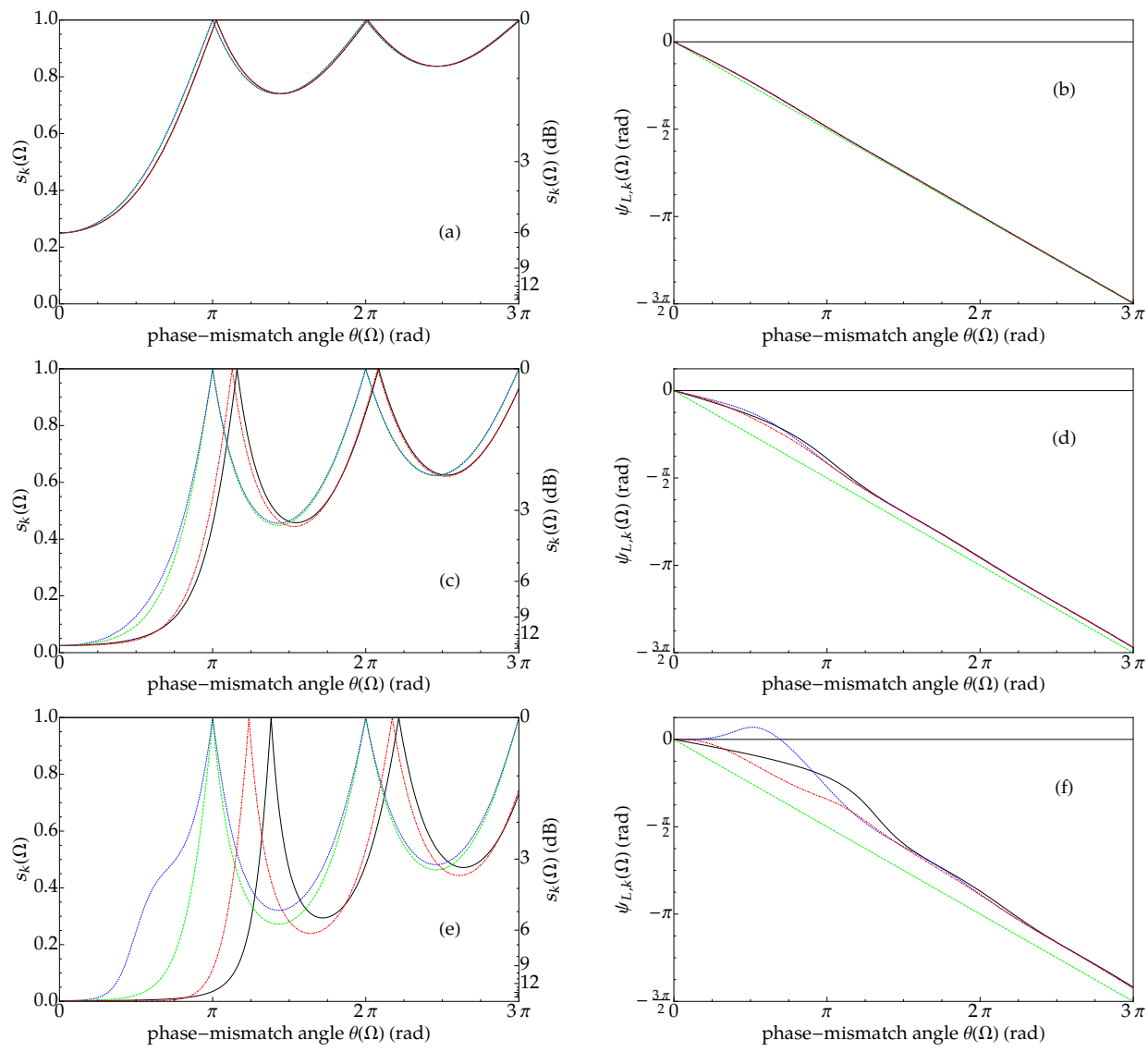

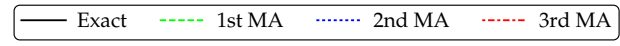

Figure 1. The squeezing spectrum $s_{k}=\exp \left(-2 r_{k}\right)(\mathrm{a}, \mathrm{c}, \mathrm{e})$ and the squeezing angle $\psi_{L, k}(\mathrm{~b}, \mathrm{~d}, \mathrm{f})$ for the first order $k=1$ (green, dashed), second order $k=2$ (blue, dotted), and third order $k=3$ (red, dotdashed) of MA compared to the exact solution (black, solid) at three levels of parametric gain $g=0.7$ (6 dB of maximal squeezing) (a,b), $g=1.8$ (16 dB of maximal squeezing) (c,d), and $g=3$ (26 dB of maximal squeezing) $(\mathrm{e}, \mathrm{f})$.

We see that at moderate squeezing, Fig. 1(a,b), the exact solution is reproduced in the first order of MA, and higher orders do not change it. Thus at such levels of squeezing the first order MA is sufficient. We can see also how increased squeezing requires higher orders of MA to yield correct behavior: In Fig. 1(c,d) the first order MA is insufficient, but the third order MA gives a satisfactory results. However, even the third order MA fails to describe the field state when the gain exponent $g$ raises towards the norm-limit of convergence, Fig. 1(e,f). 
It is notable, that the MA seems to yield improved results with increased phase mismatch. This asymptotic behavior may become clearer from Fig. 2, where higher values of the phasemismatch angle are shown.

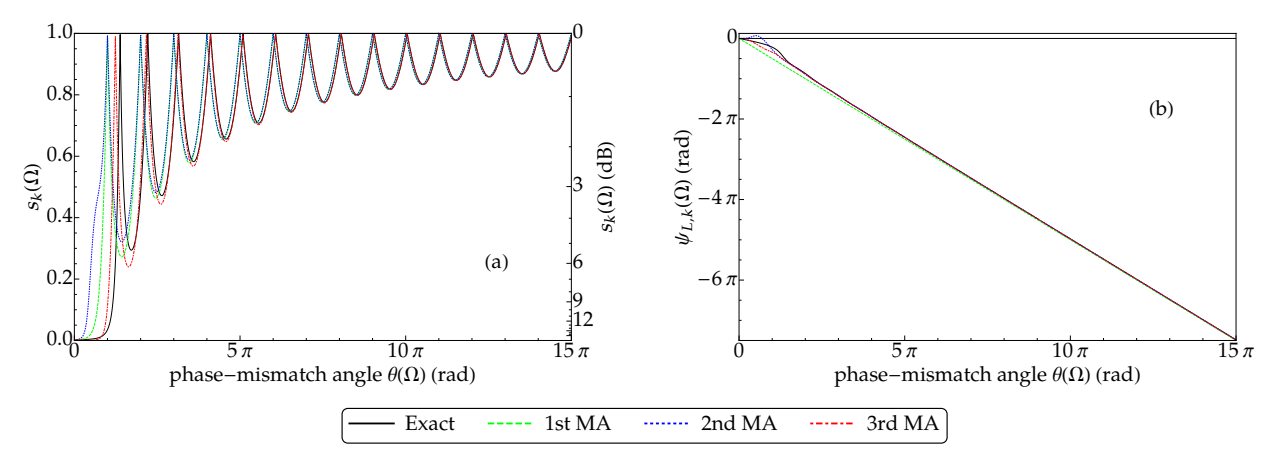

Figure 2. The squeezing spectrum $s_{k}$ (a) and the squeezing angle $\psi_{L, k}$ (b) at $g=3$ (26 dB of maximal squeezing) for the three first orders of MA compared to the exact solution for a wide range of the phase-mismatch angles $\theta$.

Here we can see an interesting property of the MA: It goes asymptotically towards the exact solution at large phase-mismatches. Additionally, let us point out that at perfect phasematching $\theta=0$ the exact solution coincides with the first order MA and the corrections from higer orders are zero.

\section{Exact upper bound}

We have seen from the previous section, that the quality of MA at high gain depends crucially on the phase mismatch. The rule of thumb for the quality of approximation is the closeness to the boundary of convergence. Thus, we can conjecture that the convergence boundary depends on the phase-mismatch angle $\theta$. In this section we show how one may achieve an exact - i.e. necessary and sufficient - upper bound for the MA that may lay above the sufficient norm criterion boundary $g<\pi$, which has been established in Ref. [12].

The symplectic matrix $\mathbf{S}_{0}$ of the exact analytic solution reads $[12,13]$

$$
\mathbf{S}_{0}(\theta, g)=\left(\begin{array}{cc}
A(\theta, g) I & B(\theta, g) P \\
B^{*}(\theta, g) P & A^{*}(\theta, g) I
\end{array}\right)
$$

where

$$
\begin{aligned}
& A(\theta, g)=e^{i \theta}\left[\cosh \left(\sqrt{g^{2}-\theta^{2}}\right)-i \frac{\theta}{\sqrt{g^{2}-\theta^{2}}} \sinh \left(\sqrt{g^{2}-\theta^{2}}\right)\right] \\
& B(\theta, g)=e^{i \theta} \frac{g e^{i \arg (\sigma)}}{\sqrt{g^{2}-\theta^{2}}} \sinh \left(\sqrt{g^{2}-\theta^{2}}\right),
\end{aligned}
$$

satisfying the unitarity condition $|A(\theta, g)|^{2}-|B(\theta, g)|^{2}=1$. This matrix can be represented as $\mathbf{S}_{0}(\theta, g)=\exp (\mathbf{M}(\theta, g))$ with the matrix

$$
\mathbf{M}(\theta, g)=\frac{\operatorname{arccosh}\left(\mathrm{A}_{\mathrm{R}}(\theta, \mathrm{g})\right)}{\sqrt{A_{R}(\theta, g)-1} \sqrt{A_{R}(\theta, g)+1}}\left(\begin{array}{cc}
i A_{I}(\theta, g) I & B(\theta, g) P \\
B^{*}(\theta, g) P & -i A_{I}(\theta, g) I
\end{array}\right),
$$


where $A_{R}(\theta, g)$ and $A_{I}(\theta, g)$ are the real and the imaginary parts of $A(\theta, g)$ respectively. The Magnus expansion corresponds to the Maclaurin series of $\mathbf{M}(\theta, g)$ in $g$. It can be shown, that the singularities of an analytical continuation $\mathbf{M}(\theta, g) \mapsto \mathbf{M}(\theta, z)$ with $z \in \mathbb{C}$, determine the radius of convergence of the Maclaurin series [17]. Thereby we can determine the exact upper bound for $g$ up to which the Magnus expansion converges. The result is displayed in Fig. 3.

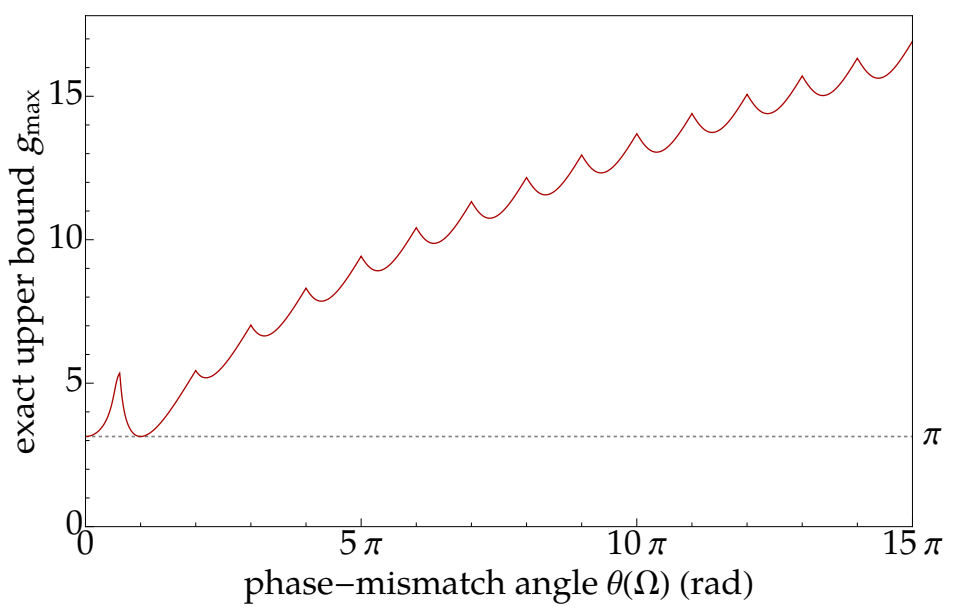

Figure 3. The exact upper bound in the gain exponent $g_{\max }$ for the convergence of the Magnus expansion as a function of the phase-mismatch angle $\theta$ (dark red). It is compared to the gain exponent $g=\pi$ which is the upper bound obtained from the spectral norm criterion (gray, dashed).

We can see that the Magnus approximation converges well beyond the limit $g<\pi$ for a wide range of $\theta$. We can also observe that the limit of convergence increases with increasing phase-mismatch. This might explain the growing quality of approximation at high phase mismatch, discussed in the previous section. Let us however stress that the above treatment has been only possible thanks to the availability of the exact solution for the considered scenario of PDC.

\section{Conclusions}

We have analyzed explicitly the dependence of the squeezing spectrum and the squeezing angle on the parametric gain in PDC with undepleted monochromatic pump. We have demonstrated that the contribution of the higher orders of the Magnus expansion is important for intermediate phase-mismatch angle only, being zero at the perfect phase-matching and negligible at a large phase mismatch. Besides, we have found the exact upper bound in gain for the convergence of the Magnus series as a function of the phase-mismatch angle and shown that for certain angles it is much higher than the value $g=\pi$, obtained in Ref. [12]. We hope that our results, obtained for the case where the exact solution exists, may be partially valid for the cases where such solution is not available, e.g. pulsed PDC, and may yield useful intuition for application of the Magnus expansion to the study of multimode squeezed light produced by PDC. 


\section{Acknowledgments}

This work was supported by the European Union's Horizon 2020 research and innovation program under grant agreement No 665148 (QCUMbER).

\section{References}

[1] U.L. Andersen, T. Gehring, C. Marquardt, G. Leuchs, Phys. Scr. 91, 053001 (2016)

[2] H. Vahlbruch, M. Mehmet, K. Danzmann, R. Schnabel, Phys. Rev. Lett. 117, 110801 (2016)

[3] R.S. Bennink, R.W. Boyd, Phys. Rev. A 66, 053815 (2002)

[4] W. Wasilewski, A.I. Lvovsky, K. Banaszek, C. Radzewicz, Phys. Rev. A 73, 063819 (2006)

[5] M.V. Chekhova, S. Germanskiy, D.B. Horoshko, G.K. Kitaeva, M.I. Kolobov, G. Leuchs, C.R. Phillips, P.A. Prudkovskii, Opt. Lett. 43, 375 (2018)

[6] A. Christ, B. Brecht, W. Mauerer, C. Silberhorn, New J. Phys. 15, 053038 (2013)

[7] N. Quesada, J.E. Sipe, Phys. Rev. A 90, 063840 (2014)

[8] N. Quesada, J.E. Sipe, Phys. Rev. Lett. 114, 093903 (2015)

[9] N. Quesada, J.E. Sipe, Opt. Lett. 41, 364 (2016)

[10] W. Magnus, Commun. Pure Appl. Math. 7, 649 (1954)

[11] S. Blanes, F. Casas, J. Oteo, J. Ros, Phys. Rep. 470, 151 (2009)

[12] T. Lipfert, D.B. Horoshko, G. Patera, M.I. Kolobov, Phys. Rev. A 98, 013815 (2018)

[13] M.I. Kolobov, Rev. Mod. Phys. 71, 1539 (1999)

[14] S.L. Braunstein, Phys. Rev. A 71, 055801 (2005)

[15] D.B. Horoshko, M.I. Kolobov, Phys. Rev. A 88, 033806 (2013)

[16] D.B. Horoshko, M.I. Kolobov, Phys. Rev. A 95, 033837 (2017)

[17] T. Lipfert, F. Krumm, M.I. Kolobov, W. Vogel, arXiv:1809.04391 